\title{
Posterior knee pain
}

\author{
S. English • D. Perret
}

Published online: 12 June 2010

(C) The Author(s) 2010. This article is published with open access at Springerlink.com

\begin{abstract}
Posterior knee pain is a common patient complaint. There are broad differential diagnoses of posterior knee pain ranging from common causes such as injury to the musculotendinous structures to less common causes such as osteochondroma. A precise understanding of knee anatomy, the physical examination, and of the differential diagnosis is needed to accurately evaluate and treat posterior knee pain. This article provides a review of the anatomy and important aspects of the history and physical examination when evaluating posterior knee pain. It concludes by discussing the causes and management of posterior knee pain.
\end{abstract}

Keywords Posterior $\cdot$ Knee $\cdot$ Pain $\cdot$ Popliteal

\section{Introduction}

The purpose of this review is to aid the clinician in evaluating and treating posterior knee pain in adults. There are several strategies to evaluate and treat knee pain. One of the most effective strategies to evaluate knee pain is based on the anatomical location of the pain. Knee pain more commonly presents in the anterior, medial, and lateral aspect of the knee and less often in the posterior aspect of the knee. The differential diagnosis for posterior knee pain is broad and includes pathology to the bones,

\section{Perret}

Department of Anesthesiology and Perioperative Care,

The University of California Irvine, Irvine, CA, USA

S. English $(\varangle) \cdot$ D. Perret

Department of Physical Medicine and Rehabilitation,

The University of California Irvine, Irvine, CA, USA

e-mail: senglish@uci.edu musculotendinous structures, ligaments, nerves, vascular components, and/or to the bursas. In order to understand the causes of posterior knee pain, the clinician needs to understand the anatomy and the important aspects of the history and physical examination when evaluating posterior knee pain. This review describes those aspects and concludes by discussing the causes and management of posterior knee pain.

\section{Anatomy and biomechanics}

The knee functions as a modified hinge joint consisting of the tibia, femur, and patella. The primary plane of motion is extension and flexion; however, when pathology is present abduction, adduction, internal and external rotations, and anterior and posterior translations may occur [1].

The distal femur and proximal tibia form the two largest points of contact in the joint. Between these two bones exists the lateral and medial menisci. These fibrocartilaginous disks contribute to joint stability by functioning as shock absorbers, increasing congruency between tibia and femur, and aiding in the distribution of synovial fluid [1]. The lateral and medial menisci differ in their sizes and attachments. The medial meniscus is smaller and attaches to the joint capsule on its entire peripheral edge, while the lateral meniscus is larger and does not attach to the capsule on the posterolateral region; this makes the medial meniscus less mobile and prone to tearing [1].

There is also a complicated network of muscles, ligaments, and other soft tissues around the knee that contributes to the structure and support of the joint. These include both passive and active stabilizers. Examples of passive stabilizers include the medial collateral ligament (MCL), lateral collateral ligament (LCL), anterior cruciate 
ligament (ACL), and posterior cruciate ligament (PCL), while examples of active stabilizers consist of the hamstrings, extensor mechanisms, and the popliteus muscle [1].

The important ligaments of the knee are the ACL, PCL, LCL, and MCL. The ACL connects the medial border of the lateral femoral condyle to the anterior aspect of the tibia, while the PCL connects the lateral border of the medial femoral condyle to the posterior aspect of the tibia. Thus, the main actions of the ACL and PCL are to prevent anterior and posterior translations, respectively. The lateral collateral ligament resists varus stress. The MCL has superficial and deep divisions that resist valgus stress [1].

The lateral collateral ligament is part of the posterolateral corner or posterior complex (PLC) of the knee. The other major components of the posterior lateral complex are the popliteus and popliteofibular ligament (PFL). The PLC resists posterior, varus, and external rotating forces [2]. Posterior laxity is most pronounced when there is also an injury to the PCL [2].

The popliteus tendon is an important cause of posterior knee pain because of its involvement in the PLC and its location on the floor of the posterior fossa. The popliteus originates from the lateral femoral condyle, the proximal fibula, and lateral meniscus and attaches to the proximal tibia superior to the soleal line [3]. The popliteus resists posterior displacement and internally rotates the tibia [4]. It is responsible for unlocking the knee during flexion from full extension [5]. It also withdraws the meniscus preventing impingement [6].

The other two sets of muscles important in posterior knee anatomy are the gastrocnemius and the hamstring complex. The gastrocnemius crosses two different joints. It originates from the posterior aspects of the femur on the medial and lateral condyles and inserts on the calcaneus [7]. The hamstring extends the hip and flexes the knee, which is important for the gait cycle [8]. The hamstring muscle group consists of the medially located semimembranosus and semitendinosus and the laterally located biceps femoris [9]. The semitendinosus and semimembranosus originate from the ischial tuberosity and insert onto the posterior medial tibia. The semimembranosus fusiform configuration and tendency to perform eccentric contraction make it susceptible to strain [7].

The biceps femoris consists of a short and long head. The long head of the biceps femoris originates from the ischial tuberosity, while the short head originates from the intermuscular septum, lateral supracondylar line, and linea aspera [8]. The biceps femoris tendon has multiple forms of attachments to the tibia on the lateral condyle, the head of the fibula, and to fascia on the leg [8]. The biceps femoris is innervated by the tibial and sciatic nerves.

It is also important to note the anatomy of the popliteal fossa when evaluating posterior knee pain. The popliteal fossa is also composed of nerves (posterior femoral cutaneous, common peroneal and tibial), vascular structures (small saphenous vein, popliteal artery and popliteal vein), bursae, lymph nodes, and fat [10]. The bursae of the knee are synovial-lined sacs that decrease the friction of moving structures [11]. Pain and swelling of the bursa is caused by inflammation, external pressure, or overuse [12].

\section{Physical examination}

Many clues in a history can aid a physician in diagnosing posterior knee pain and determine the focus of the physical examination. A detailed description of a complete knee examination is beyond the scope of this review. However, there are several key aspects of the physical examination and several examination tests that are particularly unique and important when evaluating posterior knee pain. During the initial examination, observation of the patient in the prone and standing positions should both be performed. This should be followed by active and passive range of motion of the knee in flexion, extension, and tibial rotation. The physician needs to perform resisted movements of knee flexion in tibial neutral and external tibial rotations [5]. Palpation should be performed of the joint line and of the muscle tendons of the hamstring, gastrocnemius, and popliteus. Tenderness of palpation over these tendons or muscles would indicate muscle or tendon injury. Pain or fullness in the popliteal area suggests an effusion or cyst [12].

It is important to screen for knee instability with tests that examine posterior, anterior, valgus, varus, and rotational instability. The anterior drawer and Lachman tests help diagnose ACL instability. Collateral ligaments can be isolated during valgus and varus testing by performing the examination at $30^{\circ}$ of flexion [2].

There are several tests that focus on posterior knee instability. These include the posterior sag test, posterior drawer test, and quadriceps active test. Posterior instability suggests PCL and/or PLC tear. The posterior drawer test is performed by grabbing the proximal tibia and pushing it in a posterior direction; this is compared to the degree of displacement of the unaffected side [12]. When this test is done at $90^{\circ}$ of flexion, it evaluates the PCL, while at $30^{\circ}$ of flexion, this test evaluates the PLC [2]. The posterior sag test is performed by having a supine patient flex his or her knee to $90^{\circ}$, with the feet placed flat on the table, and then relaxing his or her muscles with a focus on the quadriceps [13]. The anterior tibial plateau should remain $10 \mathrm{~mm}$ anterior to the femoral condyles [12].

The quadriceps active test is performed by having a supine patient flex his or her knee to $90^{\circ}$ with the foot fixed flat against the table. The patient then attempts to slide his 
or her foot anteriorly by contracting the quadriceps muscles. If the tibia is posteriorly displaced, the tibia will move in an anterior direction upon quadriceps contraction [13].

It is also important to assess posterolateral rotator instability. Tests include the reverse pivot shift, the dial test, and recurvatum test. In the reverse pivot shift, the patient's knee is flexed at $60^{\circ}-90^{\circ}$ and the tibia is forced into external rotation. In this position, a PCL deficient knee will be in tibial subluxation in the posterolateral direction [13]. The clinician will then extend the knee. In a posterolateral subluxed knee, the tibia will reduce at around $30^{\circ}$ of flexion and there will be a palpable shift [13]. The recurvatum test is performed by lifting the extremity by the great toe. If the knee falls into hyperextension, external rotation, and varus, then there may be a PLC injury [13].

Another test used to diagnose posterior instability is the dial test. This is helpful before surgery on a PCL deficient knee in detecting a combined injury to the PLC [14]. The dial test can clinically valuable when three posterolateral structures (popliteus tendon, PFL, and LCL) are injured or when there is combined injury to the PCL and two other posterolateral structures; however, injury to just one or two structures may be undiagnosed by use of the dial test alone [15]. The dial test is performed by grabbing the soles of both feet and applying an external rotatory force, while using the border of the medial foot as a reference against the axis of the femur [16]. A side-to-side foot-thigh angle difference of greater than $10^{\circ}$ of external rotation is considered positive [13]. The dial test can be performed either in a prone or supine position at either $30^{\circ}$ or $90^{\circ}$ of knee flexion [15]. The tibia is reduced with an anterior force when the patient is supine [17]. Performance of the dial test with the patient in prone (or when the patient is supine with an anterior force applied) is sensitive for the detection of a PLC injury in a PCL injured knee [17]. An isolated injury to the PLC can be found on examination when there is greater than $10^{\circ}$ of external rotation in the injured knee compared to the uninjured knee present at $30^{\circ}$ of flexion, but not present at $90^{\circ}$ of flexion [13].

The Apley Grind and McMurray tests are specific for the analysis of meniscal tears. When evaluating the medial meniscus, the McMurray test starts by flexing the knee to $90^{\circ}$ and externally rotating the foot. The knee is then extended, while the medial knee is palpated for a click; alternatively, the patient may report pain. When using the test to evaluate the lateral meniscus, the foot is rotated internally and the lateral knee is palpated. It should be noted that the test is only valid if the knee can be flexed past $90^{\circ}$ without pain [12].

The Apley Grind test is performed by placing the patient in a prone position with knee flexion of $90^{\circ}$. The physician then grabs the foot and applies a downward force while rotating the foot in a grinding motion. This maneuver is positive when this motion elicits pain by causing the tibia and femur to compress the meniscus [12]. A complete physical examination for posterior knee pain should also include a comprehensive musculoskeletal examination of both the back and hips, because referred pain, such as from intra-articular hip pathology, lumbar facet arthropathy, sacroiliac join dysfunction, and sacral radiculopathy, can often present with posterior knee pain. It is also important to perform a neurological examination of the limb, as peripheral neurological injury can cause knee pain or injury. The vascular system should be evaluated for potential causes of pain as well.

\section{Causes and treatment}

There is a large variety of potential causes of posterior knee pain. These include soft-tissue injuries, tendon injuries, vascular, and neurologic causes. Injuries to the soft-tissues and tendons are more common while neurologic and vascular injuries occur less frequently [18].

\section{Tendonopathy and muscle complex injuries}

Tendon injuries are associated with repetitive mechanical stresses that cause degenerative lesions [4]. The general treatment for tendonopathy injuries is surgery for complete (and possibly partial) tendon ruptures, as well as for chronic tendinopathies that have failed a 3-6-month course of conservative treatment [4].

There are three sets of muscle complexes that can typically cause posterior knee pain. They include the hamstrings, gastrocnemius, and popliteus tendon/muscle complexes. The hamstrings are frequently injured in sports that require sprinting [19]. Semimembranosus tendonopathy usually presents as an aching pain localized to the posteromedial knee with tenderness on palpation inferior to the joint [4]. Sometimes peripheral neurologic symptoms are present from external compression on adjacent structures by concomitant hematoma [18]. Some of the possible risk factors for hamstring injury include body mechanics, flexibility, balance, hamstring strength, improper warm-up, fatigue, specific activities, running techniques, and psychosocial factors [9].

There is little evidence indicating the proper management of hamstring injuries [19]. Treatments for hamstring injuries include cryotherapy, immobilization, mobilization, non-steroidal anti-inflammatory drugs (NSAIDs), electrophysical therapies, and surgery [20]. Surgical treatment in selected cases can produce beneficial results [19]. There are several studies in the literature which discuss the prevention of hamstring injuries and which comment on nonsurgical treatment options. One study evaluated the effects 
of static and dynamic stretching and of warm-up on flexibility. This study found that warm-up and static stretching increased the flexibility of the hamstring, but dynamic stretching failed to demonstrate increased flexibility [21]. Although warm up and static stretching were found to increase flexibility, it is not clear whether these actually reduce injury. In a Cochrane review, it was reported that there is insufficient evidence to conclude that an increased frequency of hamstring stretching decreases the time to recovery [22]. Other potential interventions for the rehabilitation of hamstring injury are treatments that focus on postural control, stabilization of the lumbar spine, pelvic alignment, and sacroiliac alignment [22].

Injuries of the gastrocnemius can occur at the knee [7]. Both the gastrocnemius muscle's superficial location and action across the knee and ankle make it susceptible to injury [7]. Pain in the gastrocnemius occurs during knee flexion with resistance and during calf raises [5]. Strain injuries of the gastrocnemius can be isolated or can occur in combination with other tendon or ligament injuries (such as biceps femoris, semimembranosus, soleus, PLC, or plantaris injuries) [7].

The popliteus muscle can also be a significant source of posterior knee pain. Both the muscular and tendinous aspects of the popliteus can be injured [23]. Injuries to the popliteus may promote injuries to other structures in the PLC and to ligaments of the knee. The mechanism of injury is thought to occur from direct stretch or when overused to maintain posterolateral stability [5]. Guha et al. suggested that a stable knee with posterolateral pain and hemarthrosis on exam indicates a rupture of the popliteus tendon [24]. Other signs of popliteus muscle injury include pain while weight bearing with the knee flexed at $15^{\circ}-30^{\circ}$ or pain during the initial swing phase of the gait cycle [4]. The diagnosis of popliteus tendon injury can be difficult to make and an examination involving analysis of the gait, laxity of the knee, an evaluation for effusion, and for posterolateral knee tenderness should be performed [25]. However, no one specific test can reliably reveal the degree of injury [25]. For instance, while most popliteus injuries involve the musculotendinous junction and the tendon proper, $<50 \%$ of the tendon is visualized during arthroscopic inspection, and the musculotendinous junction and femoral insertion are completely excluded [25]. This demonstrates the inadequacy of performing an arthroscopic examination by itself. The treatment plan for an isolated popliteus tendon rupture has not been formalized. It is known that a knee stable on examination may be successfully managed conservatively [24]. However, surgical options are also possible. Murray et al. advocate a differential strategy based on age [26]. In the elderly patient, surgery is indicated only when the knee is locked; in this case, a simple resection can be done without causing functional deficits [26]. In the younger patient, a more aggressive surgical approach is recommended [26].

Popliteus rupture commonly occurs in combination with other PLC injuries [26]. An individual with a PLC injury may have external rotation and varus instability of the knee with swelling and point tenderness. Diagnosis of PLC injuries can be assisted by arthroscopy and MRI [16]. MRI displays a detailed evaluation of the PLC [27]. Arthroscopy, while limited in diagnosing an isolated popliteal injury, is valuable in the diagnosis of complex injuries. Arthroscopy allows visualization and confirmation of associated cruciate, meniscal, and chondral injuries involving areas that could have been hidden on MRI [16]. Posterolateral corner injuries can be managed both operatively and non-operatively. However, it has been suggested that operative treatments have improved functional results [28].

\section{Nerve injury}

Injuries to the posterolateral aspect of the knee can often cause nerve injury to the common peroneal nerve. This was demonstrated in a case report of a tennis player with a ruptured popliteal muscle that caused palsy to the tibial nerve [23]. Consequently, posterolateral injury work-up should include a complete neurological examination [24].

\section{Ligamentous injury}

Injuries to the posterolateral structures are rarely isolated [2]. A PLC injury frequently occurs along with PCL and ACL injuries. Failure to recognize a PLC injury when treating an ACL or PCL injury can cause chronic instability [29]. This may also result in a tendency for cruciate graft failures [29]. This concept was illustrated in a cadaveric study: Sectioning of the PCL caused posterolateral structures to suffer increased forces throughout flexion [2]. Therefore, during reconstruction of the knee, it is important to identify damage in the PLC to prevent graft failure and restore stability [16]. It should be noted that patients undergoing ACL/PCL and PLC repair or reconstruction have similar outcomes [28].

The classic PCL injury is from a motor vehicle accident where a posteriorly directed force from the dash board onto a flexed knee occurs; this is a so-called dash board injury [30, 31]. A posterior drawer test can usually help identify PCL injuries. In addition to PCL injuries being associated with posterolateral corner injuries, as described above, the literature suggests that a deficiency in the PCL causes increased contact pressure in the tibiofemoral and patellofemoral joints, damage to the articular cartilage, and meniscal tears [32]. 
Both operative and non-operative treatment strategies have been used for PCL injuries. However, the long-term results of non-operative treatment are unknown and may be associated with knee instability [33]. A complete examination to determine the cause of injury before deciding upon a management strategy is required [32]. A finding of articular damage is important in determining if surgery is necessary [32]. For improved outcomes, surgical reconstruction is indicated in severe posterior instability and when injury occurs to multiple ligaments [33]. Hence, when making treatment decisions on PCL injuries, the clinician should consider knee stability, timing, pain severity, associated injuries, MRI findings, and life style [33].

The ACL is a rare cause of posterior knee pain. Motmans et al. describe a case report where a patient who presented with pain in the posterior knee was found to have mucoid degeneration of the ACL [34]. In this case, the patient underwent arthroscopy and the hypertrophied tissue was debrided [34]. The authors found that this treatment relieved the patient's pain and did not decrease stability [34]. However, this is a single case report and larger studies need to be performed.

\section{Meniscal injury}

A meniscal tear can cause a catching sensation in the knee and rarely presents as posterior knee pain. This is particularly true for medial meniscus tears of the posterior horn [35]. There are three classic diagnostic exams described in the physical examination literature. These include: joint line tenderness, Apley's maneuver, and McMurray's maneuver. A meta-analysis found that these common tests for meniscal lesions demonstrate findings of low-to-moderate diagnostic accuracy [36]. However, a meta-analysis also found joint line tenderness to be superior to Apley's and McMurray's maneuvers in diagnostic utility [36]. With this questionable accuracy of special tests performed during the physical examination, it should be noted that when performing an arthroscopy based on a clinical diagnosis of a posterior horn lateral meniscal tear, other pathologies need to be ruled out, especially if the tear in the meniscus is not confirmed [37]. In one case report, the clinical diagnosis of meniscus tear was not confirmed during arthroscopy, but instead the presence of calcific deposits on the popliteus tendon was noted as a potential source of the pain [37]. These deposits were debrided arthroscopically and there was pain resolution [37].

Bone injury

Bone pain can rarely cause posterior knee pain. Bone pathologies include degenerative bone disease, tibial stress fractures, and bone tumors. Bone tumors are a rare cause of pain that can be difficult to diagnose. Of 667 knee tumors studied at an institution in Italy, 25 (3.7\%) were previously erroneously treated with an intraarticular procedure secondary to misdiagnosis [38]. The authors found that the most frequent cause for inaccurate diagnoses was poor radiographs and not questioning the original diagnosis when symptoms continued [38]. Overall treatment may be adversely affected by postponing the time to the accurate diagnosis and treatment and by inappropriately performing an invasive procedure that may even lead to tumor extension, contamination of margins, and/or cause tumor progression [38]. The treatment of bone tumors includes amputation, limb salvage surgery, chemotherapy, and radiation therapy [18].

Besides bony tumors, some other tumors can also cause knee pain. These tumors include osteochondromas, osteosarcomas, chondroblastomas, endochondromas, synovial chondromatosis, and pigmented villonodular synovitis [18]. Recently, an ngiomyomatous hamartoma has been described as causing posterior knee pain. This angiomyomatous hamartoma was in a single popliteal lymph node that caused posterior knee pain [39].

Knee cysts and bursal injury

There are numerous bursae around the knee that act to decrease friction between moving structures [40]. Injury to bursae is usually the result repetitive motions and will elicit pain and tenderness. Cystic lesions of the knee can be caused from a diverse group of entities from benign etiologies to complications of arthritis, infection, and malignancy [12]. MRI is the best choice for describing cystic lesions of the knee because it confirms the presence of a cyst, it demonstrates its anatomic relationship with surrounding structures, and it helps delineate any associated pathology [40].

The classic cystic lesion causing posterior knee pain is the Baker's (or popliteal) cyst. A Baker's cyst is caused either by a herniation of the synovial membrane through the posterior capsule or by an escape of fluid through an anatomic bursa next to semimembranosus or gastrocnemius [6]. It is thought that Baker's cysts are usually caused by a combination of weakness and internal mechanical pathology resulting in increased intra-articular pressure [11]. Popliteal cysts are associated with a variety of internal derangements that include arthritis, ligament tears, meniscal tears, sarcoidosis, and even lymphomas [40]. It is hypothesized that Baker's popliteal cysts cause symptoms either by compression of adjacent structures or occasionally due to rupture or leakage [41]. Most Baker's cysts can be easily noted on physical examination because the cyst can be palpated between the semimembranosus and the medial head of the gastrocnemius [41]. 
Occasionally, it may be difficult to differentiate a popliteal cyst from other causes of posterior knee pain. The differential of a popliteal cyst includes an arterial popliteal aneurysm or toruosity, adipose tissue, tumor, or deep vessel thrombosis [11]. There are many diagnostic techniques to evaluate such a cyst including radiography, ultrasound, arthrography, MRI, CT, and MR arthrography; all of these can be helpful in the evaluation process [42]. Ultrasound has replaced arthrography as the usual initial assessment tool [41]. However, overall, the best imaging for assessment of a popliteal cyst is MRI because it allows for precise localization of the cyst and identifies any internal derangements [41]. Therefore, ultrasound is used to confirm a cyst, evaluate the cysts internal structures, exclude other lesions, and assess its relationship to other structures; MRI is used for a more complete evaluation of the internal knee structures [42]. In some cases, the final diagnosis can be established by aspiration of the mass, but this is rarely done. When solid components are present, a synovial tumor should be rule out [41].

It is important to properly diagnose a Baker's cyst to prevent complications and to determine the best management strategy [41]. Complications of a Baker's cyst include rupture, infection, compression of adjacent structures, and hemorrhage. Compression of adjacent structures can result in nerve entrapment, deep venous thrombosis, and ischemia [40]. Large cysts can lead to difficulty performing knee flexion and to reduced mobility [40]. The cyst may also rupture and spill synovial fluid into the surrounding tissues [43]. This may clinically mimic acute thrombophlebitis [43]. This usually can be differentiated by using ultrasound or venography. An infection is also a complication of a Baker's cyst and can be associated with septic arthritis [43]. This should be suspected in patients with a systemic infection and a popliteal cyst with local signs of infection [43]. This has been illustrated in a case report of a patient with unilateral lower extremity swelling and pain as well as in another case report where septic arthritis was induced by a corticosteroid injection which was complicated by a pyogenic Baker's cyst $[43,44]$. There are many treatment options for a Baker's cyst. The treatment choice depends on the causative etiology, as there is a high recurrence rate if the underlying condition is not treated. Treatment can include aspiration or surgical excision; however, spontaneous resolution is common without any treatment [6]. When the cyst does not resolve after treating of the underlying condition, surgical treatment may be performed [42]. When treating osteoarthritis associated with Baker's cyst, an intra-articular corticosteroid injection provides a reduction in the dimension and wall thickness of the Baker's cyst [45]. The authors hypothesized that the steroid not only reduced intraarticular knee inflammation, but also reduced inflammation on the synovial membrane of the cyst by migration of the steroid into the cyst [45].

Sclerotherapy is also one of the treatment options for Baker's cyst. In one case report, a 52-year-old patient with a meniscus tear and a Baker's cyst was treated with three sessions of sclerotherapy and had resolution of the Baker's cyst [46]. If surgical excision is being considered, an arthroscopic examination should first be performed and any pathologic condition treated [42].

A ganglion is also a cystic lesion. A ganglion does not have a synovial lining, but instead has a wall that is thick with fibrous connective tissue [40]. Ganglion cysts around the knee can be extra-articular, intra-articular, intra-osseous, and periosteal [40]. Intra-articular ganglion cysts arise from the cruciate ligaments of the ACL more frequently than PCL [40]. Ganglions cause local swelling, pain and signs of compression. Ganglion presence in the popliteus sheath may cause pain [3, 47]. Ganglion cysts can be treated by puncture, aspiration, excision, or corticosteroid injection [11].

Other neurological and vascular injuries

There are several neurological and vascular causes of knee pain. These include popliteal artery entrapment syndrome, aneurysms, and deep venous thrombosis (DVT). Popliteal artery entrapment presents with knee pain, swelling, and ischemic changes. Popliteal artery entrapment is usually caused by abnormal anatomy between the muscles and arteries [48]. However, it can also occur in the absence of an abnormal anatomy [49]. Popliteal artery entrapment may present bilaterally [50]. The diagnosis may be established with MRI or CTA [51]. Angiography is also helpful in the diagnosis. When intervention is indicated in an operative candidate, the preferred treatment to prevent ischemia is popliteal artery release with or without vein bypass [52].

There is minimal literature available on neurological causes of posterior knee pain as there are few case reports, randomized control trials, or meta-analyses that discuss the neurological causes of posterior knee pain. However, referred pain has been implicated as a cause of posterior knee pain. The patellofemoral joint and lumbar spine may both refer pain to the posterior knee [5]. Pain can also be caused by entrapment of nerves in the popliteal fossa.

\section{Conclusions}

There are broad differential diagnoses for causes of posterior knee pain. A physician needs to be aware of the common causes, such as musculotendinous injury or Baker's cyst, and also of the rare causes of knee pain such 
as bone tumors and damage to peroneal nerve. An accurate understanding of knee anatomy, the physical examination, and of the differential diagnosis is needed to accurately evaluate and treat patients with posterior knee pain.

Open Access This article is distributed under the terms of the Creative Commons Attribution Noncommercial License which permits any noncommercial use, distribution, and reproduction in any medium, provided the original author(s) and source are credited.

\section{References}

1. Skinner H. Current diagnosis and treatment in orthopedics. 4th ed. New York: McGraw-Hill; 2006.

2. Frank J, Youm T, Meislin R, et al. Posterolateral corner injuries of the knee. Bull NYU Hosp Jt Dis. 2007;65(2):106-14.

3. Blake S, Treble N. Popliteus tendon tenosynovitis. Br J Sports Med. 2005;39:42.

4. Boden B, editor. Tendon injuries basic science and clinical medicine. London: Springer; 2005.

5. Brukner P, Khan K, editors. Clinical sports medicine. 3rd ed. Australia: McGraw-Hill; 2007.

6. Marx J. Rosen's emergency medicine. 6th ed. Philadelphia, PA: Mosby Elsevier; 2006.

7. Bencardino J, Rosenberg Z, Brown R, et al. Traumatic musculotendinous injuries of the knee: diagnosis with MRI. RadioGraphics. 2000;20:S103-20.

8. Koulouris G, Connell D. Hamstring muscle complex: an imaging review. RadioGraphics. 2005;25:571-86.

9. Hoskins W, Pollard H. The management of hamstring injury. Part I: issues in diagnosis. Man Ther. 2005;10:96-107.

10. Woodley S, Mercer S. Anatomy in practice: the popliteus muscle. N Z J Physiother. 2006;34(1):25-9.

11. Beaman F, Peterson J. MR imaging of cysts, ganglia, and bursae about the knee. Radiol Clin N Am. 2007;45:969-82.

12. McPhee S, Papadakis M, Gonzales R, et al. Current medical diagnosis and treatment. 48th ed. New York: McGraw-Hill; 2009.

13. Lubowitz J, Bernardini B, Reid J, et al. Comprehensive physical examination for instability of the knee. Am J Sports Med. 2008;36(3):577-594.

14. Jung YB, Lee YS, Jung HJ, et al. Evaluation of posterolateral rotatory knee instability using the dial test according to tibial positioning. J Arthrosc Relat Surg. 2008;25(3):257-61.

15. Bae J, Choi I, Suh S, et al. Evaluation of the reliability of the dial test for posterolateral rotatory instability: a cadaveric study using an isotonic rotation machine. $\mathrm{J}$ Arthrosc Relat Surg. 2008;24(5):593-8.

16. Davies H, Unwin A, Aichroth $\mathrm{P}$, et al. The posterolateral corner of the knee anatomy, biomechanics and management of injuries. Int J Care Inj. 2004;35:68-75.

17. Strauss E, Ishak C, Inzerillo $C$, et al. Effect of tibial positioning on the diagnosis of posterolateral rotatory instability in the posterior cruciate ligament-deficient knee. Br J Sports Med. 2007;41: 481-5.

18. Muche J, Lento P. Posterior knee pain and its causes. Phys Sports Med J. 2004;32:4.

19. Lempainen L, Sarimo J, Mattila K, et al. Distal tears of the hamstring muscle: review of the literature and our results of surgical treatment. Br J Sports Med. 2007;41:80-3.

20. Hoskins W, Pollard H. Hamstring injury management. Part 2: treatment. Man Ther. 2005;10:180-90.
21. O'Sullivan K, Murray E, Sainsbury D, et al. The effect of warmup, static stretching and dynamic stretching on hamstring flexibility in previously injured subjects. BioMed Cent Musculoskelet Disord. 2009;10:37.

22. Mason DL, Dickens VA, Vail A. Rehabilitation for hamstring injuries: review. Cochrane Database Syst Rev. 2007;1:CD004 575.

23. Ortiguera C, Bremner B, Peterson J, et al. Popliteus strain causing tibial nerve palsy with a permanent partial deficit. Am J Sports Med. 2006;34:7.

24. Guha A, Gorgees K, Walker D, et al. Popliteus tendon rupture: a case report and review of the literature. Am J Sports Med. 2003;37:358-60.

25. Fineberg M, Duquin T, Axelrod J, et al. Arthroscopic visualization of the popliteus tendon. J Arthrosc Relate Surg. 2008;24(2):174-7.

26. Murray J, Grundy J, Collins I, et al. Spontaneous rupture of the popliteus tendon in a 74-year-old woman and review of the literature. J Arthrosc Relate Surg. 2004;20(8):860-4.

27. Pacholke D, Helms C. MRI of the posterolateral corner injury: a concise review. J Magn Reson Imaging. 2007;26:250-5.

28. Levy B, Dajani K, Whelan D, et al. Decision making in the multiligament-injured knee: an evidence-based systematic review. J Arthrosc Relat Surg. 2009;25(4):430-8.

29. Vinson E, Major N, Helms C, et al. The posterolateral corner of the knee. Am J Roentgenol. 2008;90:449-58.

30. Colvin A, Meislin R. Posterior cruciate ligament injuries in the athlete: diagnosis and treatment. Bull NYU Hosp Jt Dis. 2009;67(1):45-51.

31. Koulouris G, Connell D. Hamstring muscle complex: an imaging review. RadioGraphics. 2005;25:3.

32. Grassmayr M, Parker D, Coolican M, et al. Posterior cruciate ligament deficiency: biomechanical and biological consequences and the outcomes of conservative treatment-a systematic review. J Sci Med Sports. 2008;11:433-43.

33. Chen $\mathrm{CH}$. Surgical treatment of posterior cruciate ligament injury. Chang Gung Med J. 2007;30:480-92.

34. Motmans R, Verheyden F. Mucoid degeneration of the anterior cruciate ligament. Knee Surg Sports Traumatol Arthrosc. 2009; 17:737-40.

35. Edell, D. Posterior knee pain: several common possibilities. Athl Advis. 2005;10:6.

36. Meserve B, Cleland J, Boucher T, et al. A meta-analysis examining clinical test utilities for assessing meniscla injury. Clin Rehabil. 2008;22:143-61.

37. Tennent TD, Gordia VK. Arthroscopic management of calcific tendonitis of the popliteus tendon. J Arthrosc Relate Surg. 2003;19:4.

38. Muscolo D, Ayerza M, Makino A, et al. Tumors about the knee misdiagnosed as athletic injuries. J Bone Jt Surg. 2003;85:120914.

39. Mauro C, McGough R, Uma Rao, et al. Angiomyomatous hamartoma of a popliteal lymph node: an unusual cause of posterior knee pain. Ann Diagn Pathol. 2008;12:372-4.

40. Marra M, Crema M, Chung M, et al. MRI features of cystic lesions around the knee. Knee. 2008;15:423-38.

41. Torreggiani $\mathrm{W}, \mathrm{Al}-\mathrm{Ismail} \mathrm{K}$, Nunk $\mathrm{P}$, et al. The imaging spectrum of Baker's (popliteal) cysts. Clin Radiol. 2003;57:681-91.

42. Fritschy D, Fasel J, Imbert J, et al. The popliteal cyst. Knee Surg Sports Traumatol Arthrosc. 2006;14:623-8.

43. Drees C, Lewis T, Sherif M. Baker's cyst infection: case report and review. Clin Infect Dis. 1999;29:276-8.

44. Corten K, Vandernneucker H, Reynders P, et al. A pyogenic, ruptured Baker's cyst induced by arthroscopic pressure pump irrigation. Knee Surg Sports Traumatol Arthrosc. 2009;17:266-9. 
45. Acebes J, Sanches-Pernaute O, Diaz-Oca A, et al. Ultrasonographic assessment of Baker's cysts after intra-articular corticosteroid injection in knee osteoarthritis. J Clin Ultrasound. 2006;34:3.

46. Centeno C, Schultz J, Freeman M, et al. Sclerotherapy of Baker's cyst with imaging confirmation of resolution. Pain Phys J. 2008;11:257-61.

47. Weber D, Friederich N, Nidecker A, et al. Deep posterior knee pain caused by a ganglion of the popliteus tendon: a case report. Knee Surg Sports Traumatol Arthrosc. 1996;4:157-9.

48. Ikiz Z, Ucerler H, Ozgur Z, et al. Anatomic variations of popliteal artery that may be a reason for entrapment. Surg Radiol Anat. 2009; 48 .
49. Pillai J, et al. A current interpretation of popliteal vascular entrapment. J Vasc Surg. 2008;48:61S-5S.

50. Shen J, Abu-Hamad G, Makaroun M, et al. Bilateral asymmetric popliteal entrapment syndrome treated with successful surgical decompression and adjunctive thrombolysis. Vasc Endovasc Surg J. 2009;43:395-398.

51. Housseini A, Maged I, Abdel-Gawad A, et al. Popliteal artery entrapment syndrome. J Vasc Surg. 2009;49:1056.

52. Gourgiotis S, Aggelakas J, Salemis N, et al. Diagnosis and surgical approach of popliteal artery entrapment syndrome: a retrospective study. Vasc Heath Risk Manag. 2008;4(1):83-8. 